\title{
Cutting brittle rods using elastic wave propagation
}

\author{
P. Šidlof ${ }^{a}$, M. Pustka ${ }^{a, *}$, Z. Braier ${ }^{a}$ \\ ${ }^{a}$ VÚTS, a. s., Svárovská 619, 46001 Liberec XI, Czech Republic \\ Received 1 June 2020; accepted 3 May 2021
}

\begin{abstract}
For the sake of intended industrial applications, the stress wave propagation in thin rods of brittle material is measured and explored. Based on the known theory of a non-dispersive wave propagation in rods, a numerically efficient method for the calculation of wave propagation and reflection is presented. The effects of an impact applied to a thin rod are tested and measured on a test bench. The results of these measurements carried out on glass capillary tubes are successfully compared with theoretical results and they confirm the possibility of brittle rod cutting by means of elastic waves with the required fracture surface quality.
\end{abstract}

(C) 2021 University of West Bohemia. All rights reserved.

Keywords: thin rod, brittle material, longitudinal elastic wave propagation, strain gauge measurement, cutting method

\section{Introduction}

The glass blanks for the quality seed bead and chaton production are manufactured by separating small-diameter tubes or rods into short pieces (so-called preforms). The drawback of these common separating methods, for example chopping or breaking [10], is a low fracture surface quality and the preforms must be further finished, e.g., by grinding for improvement. The pursuit of a quality surface achievement commonly increases production efforts and reduces their efficiency.

For these reasons a new cutting technology based on an elastic wave propagation in a thin rod [12] was developed and experimentally verified. The principle of separation is based on the well-known Hopkinson pressure bar technique [5]. The compressive stress wave, excited, e.g., by a number of harmonic force periods or by a force pulse at the rod end, is reflected back as a tensile wave at the opposite free end. For a rod of brittle material having much higher compressive than tensile strength, the separation of the end section may occur by virtue of induced tensile stress. The procedure has recently been used, e.g., for determining the tensile strength of concrete [8] and ceramics [4] or to study the viscoelastic wave propagation along sandstone bars [11] by means of spalling tests. If the rod end section to be separated is weakened by a circumferential notch, the fracture occurs at the notch position and the fracture surface is of a high flatness, perpendicular to rod axis and without chips. This method allows the making of quality preforms with high production rate and reproducibility.

The paper deals with both theoretical and experimental analysis of the stress wave propagation in brittle rods to verify the applicability of the separation method. The one-dimensional elastic wave propagation theory turns out to be sufficiently accurate for these purposes. The propagation of two stress shapes (harmonic wave packet and pulse wave) considered for the end

\footnotetext{
*Corresponding author. Tel.: +420 485302 223, e-mail: martin.pustka@ vuts.cz. https://doi.org/10.24132/acm.2021.624
} 
separation is modelled. A test bench with an air gun is used to perform an impulse loading of glass capillary tubes with and without a notch and to record wave histories by means of a strain gauge measurement. The strain histories are finally compared with the theoretical non-dispersive wave propagation.

\section{Wave propagation in thin prismatic rods}

\subsection{Theoretical model}

In a high strain-rate loading, a solution to the longitudinal impact of a rod is generally threedimensional and the geometrical [6] and material [1] dispersion effects should be taken into account. Assuming that the ratio of rod diameter to length is small, only a short time interval of the end separation is analyzed and dispersion and attenuation effects are neglected. Then, we can apply the elementary theory of a wave propagation in thin prismatic rods [6]. This theory assumes only deformations in the axial direction and neglects the transverse deflections. The governing equation of motion is a linear partial differential equation

$$
\frac{\partial^{2} u}{\partial x^{2}}=\frac{1}{c_{0}^{2}} \frac{\partial^{2} u}{\partial t^{2}}, \quad c_{0}=\sqrt{\frac{E}{\rho}},
$$

where $c_{0}$ is the velocity of a longitudinal wave propagation in a thin rod, $E$ is Young's modulus and $\rho$ is the density. The equation of motion has a general solution demonstrating that the waves propagate in both rod directions with the velocity $c_{0}$ without a distortion. In our case we consider the solution [3] corresponding to the unit force (Heaviside step function) applied to the rod end. The case of the general force waveform can subsequently be derived by using the convolution theorem.

Let us assume a free rod of constant cross-section $S$ and length $L$, loaded at one end (at position $x=L$ ) at time $t=0$ by a unit step force $F$, as shown in Fig. 1 .

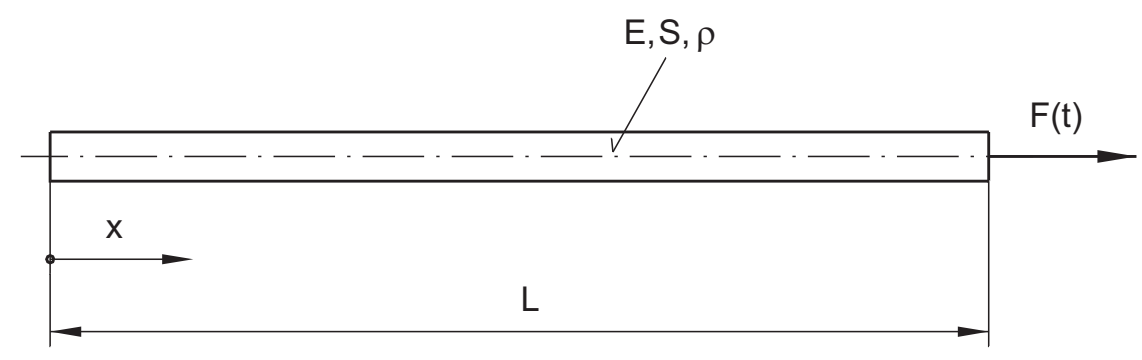

Fig. 1. Thin elastic rod loaded by a unit step force

The boundary conditions have the form

$$
\frac{\partial u(0, t)}{\partial x}=0, \quad E S \frac{\partial u(L, t)}{\partial x}=F H(t)
$$

where $H(t)$ is Heaviside step function. Applying the Laplace transform to the equation of motion (1) gives the ordinary differential equation

$$
\frac{\partial^{2} \bar{u}}{\partial x^{2}}=\frac{s^{2}}{c_{0}^{2}} \bar{u}
$$


with a solution

$$
\bar{u}(x, s)=A \cosh \left(\frac{s}{c_{0}} x\right)+B \sinh \left(\frac{s}{c_{0}} x\right),
$$

where $A, B$ are integration constants. The transformed boundary conditions (2) for the problem are

$$
\frac{\partial \bar{u}(0, s)}{\partial x}=0, \quad E S \frac{\partial \bar{u}(L, s)}{\partial x}=\frac{F}{s} .
$$

Substituting (4) into (5) we obtain the transformed solution

$$
\bar{u}(x, s)=\frac{F c_{0}}{E S} \frac{\cosh \left(\frac{s}{c_{0}} x\right)}{s^{2} \sinh \left(\frac{s}{c_{0}} L\right)} .
$$

Taking the inverse Laplace transform of (6), we arrive at the final solution in the form

$$
u(x, t)=\frac{F}{2 S L \rho} t^{2}+\frac{F}{2 E S L}\left(x^{2}-\frac{L^{2}}{3}\right)-\frac{2 F L}{E S \pi^{2}} \sum_{n=1}^{\infty} \frac{(-1)^{n}}{n^{2}} \cos \left(\frac{n \pi x}{L}\right) \cos \left(\frac{n \pi c_{0} t}{L}\right) .
$$

There are three terms on the right-hand side of (7): a uniformly accelerated motion of a solid rod caused by a force $F$, a time-independent rod elongation resulted from a rod elements continuous load by inertial forces and a non-stationary motion caused by a propagating stress wave. The last component in the form of infinite series could be expressed by the polylogarithm $\mathrm{Li}_{2}(x)$ (Jonquiere's function) [14] as

$$
\frac{F L}{2 E S \pi^{2}}\left[\operatorname{Li}_{2}\left(-\mathrm{e}^{-\frac{\mathrm{j} \pi\left(c_{0} t-x\right)}{L}}\right)+\mathrm{Li}_{2}\left(-\mathrm{e}^{\frac{\mathrm{j} \pi\left(c_{0} t-x\right)}{L}}\right)+\operatorname{Li}_{2}\left(-\mathrm{e}^{-\frac{\mathrm{j} \pi\left(c_{0} t+x\right)}{L}}\right)+\operatorname{Li}_{2}\left(-\mathrm{e}^{\frac{\mathrm{j} \pi\left(c_{0} t+x\right)}{L}}\right)\right] .
$$

Using Hooke's law $\sigma=E \frac{\partial u}{\partial x}$ we can get the longitudinal stress $\sigma(x, t)$ in a rod

$$
\begin{aligned}
\sigma(x, t)= & \frac{F x}{S L}+\frac{\mathrm{j} F}{2 \pi S}\left[\ln \left(1+\mathrm{e}^{-\frac{\mathrm{j} \pi\left(c_{0} t-x\right)}{L}}\right)-\ln \left(1+\mathrm{e}^{\frac{\mathrm{j} \pi\left(c_{0} t-x\right)}{L}}\right)-\right. \\
& \left.\ln \left(1+\mathrm{e}^{-\frac{\mathrm{j} \pi\left(c_{0} t+x\right)}{L}}\right)+\ln \left(1+\mathrm{e}^{\frac{\mathrm{j} \pi\left(c_{0} t+x\right)}{L}}\right)\right] .
\end{aligned}
$$

The resulting stress wave has a rectangular profile and when it reaches the end $(x=0)$, there is a stress $\frac{F}{S}$ in the entire rod. The wave is reflected at a free boundary with an opposite phase, interferes with a propagating wave and reduces the original stress gradually to zero. When the wave reaches the origin with acting force $F$, the stress in the entire rod is zero, but the rod now acquires the velocity corresponding to the original deformation energy. The wave is then reflected with the opposite (original) phase and the process repeats itself periodically.

In the case of loading the rod with a general force $F_{1}(t)$, the resulting response $u_{1}(x, t)$ can be obtained from the solution $u(x, t)$ using the convolution theorem. As the response $u(x, t)$ is derived for a unit step function $H(t)$, the identity for the derivative of convolution is applied [2] and the convolution integral has a form

$$
u_{1}(x, t)=\frac{\mathrm{d}}{\mathrm{d} t} \int_{0}^{t} F_{1}(\tau) u(x, t-\tau) \mathrm{d} \tau .
$$

In the following, the theoretical model is used to study the propagation of a stress wave in a long rod. Two stress excitation methods are analyzed, namely the application of harmonic force periods and the application of a force pulse. 


\subsection{Wave packet reflection at a rod free end}

In the first case, we suppose a stress wave packet in the rod excited by a number of harmonic force periods with a defined waveform. The stress wave can be generated virtually, e.g., by means of an ultrasonic transducer with a high operating frequency [9] attached to the rod end. The scheme of wave excitation shown in Fig. 2 corresponds to the orientation of the theoretical model (Fig. 1), the wave excited at the right end starts to propagate to the left free end.

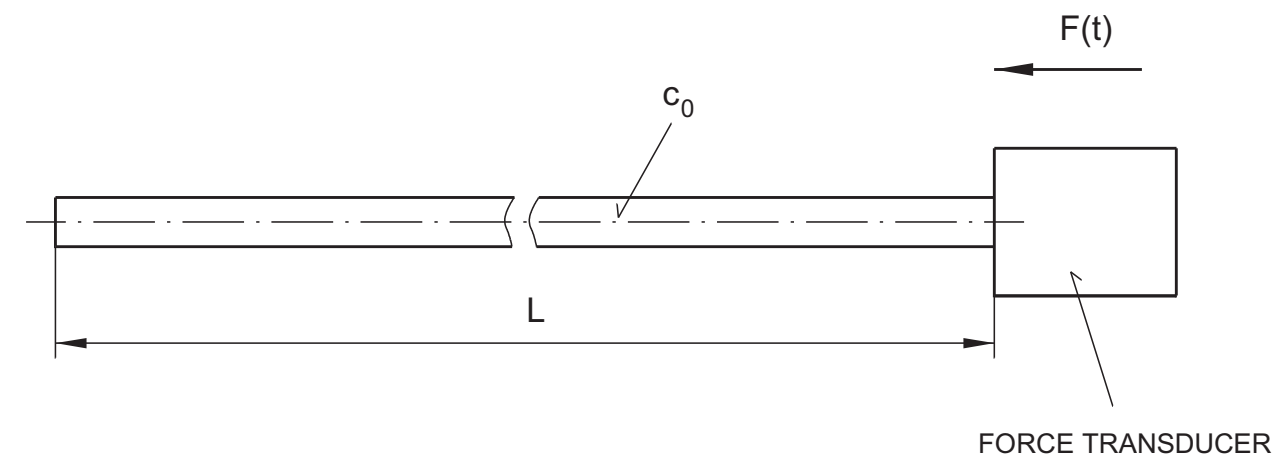

Fig. 2. Scheme of stress wave excitation by a number of harmonic force periods

The proposed stress wave packet has four harmonic periods with a modified starting part followed by a steady-state part (see Fig. 3a, the wavefront is on the left). Both parts have a length of $2 \lambda$, the amplitude of the steady-state part was selected equal to the unity. The starting part was created by multiplying the sine course by the function $k_{1}\left[1-\cos \left(k_{2} t\right)\right]$ with properly selected parameters $k_{1}, k_{2}$.

The maximal amplitudes of the harmonic stress waveform are in the distance of the wavelength $\lambda=c_{0} / f$, where $f$ is the operating frequency of the force transducer. To analyze the relation of the wavelength $\lambda$ to the length of separated preform, we express the travel of the wave packet by the relative displacement $X_{1}=c_{0} t / \lambda$, where $t$ is time. Its value represents the distance travelled by the wavefront measured in units of wavelength $\lambda$ (a wavefront travel equal to $\lambda$ corresponds to $X_{1}=1$ ).

Fig. 3 presents a series of propagating wave profiles near the rod left end with a uniform wavefront shift of one wavelength. In Fig. 3a the wavefront of the left-propagating wave reached the origin $X_{1}=0$ at the rod left end. Figs. 3b-e illustrate the combination of the incident wave and the wave reflected from the free boundary. It is obvious that the positive (tensile) stress with double amplitude occurs twice in position $X_{1}=0.25$ (1/4-wavelength) due to the interference. Fig. $3 e$ (distance $4 \lambda$ ) depicts the wave after the complete reflection, which will continue travelling to the right with an unaltered profile but with an opposite phase.

The wave profiles near the left rod end are shown in Fig. 4 for the wavefront distance $X_{1}$ from 0 to 4.5 with a step of 0.015 . The tensile stress has a double amplitude at positions $X_{1}=0.25$ and 0.75 (1/4- and 3/4-wavelength), for $X_{1}=1.25$ (5/4-wavelength) is the maximum tensile (positive) amplitude equal to 1.316 . In all other positions of $\lambda / 4$-multiples the maxima have only a unit amplitude.

These results indicate that the required length of the preform must correspond to a distance of $\lambda / 4$. For a preform with a common length of $5 \mathrm{~mm}$ and a longitudinal wave velocity in glass of $5200 \mathrm{~m} / \mathrm{s}$ we have a wavelength of $\lambda=20 \mathrm{~mm}$. The operating frequency of the force transducer $f$ is then $260 \mathrm{kHz}$, which is relatively high for practical application. The total length of the wave packet shown in Fig. 3 would be then $80 \mathrm{~mm}$. 

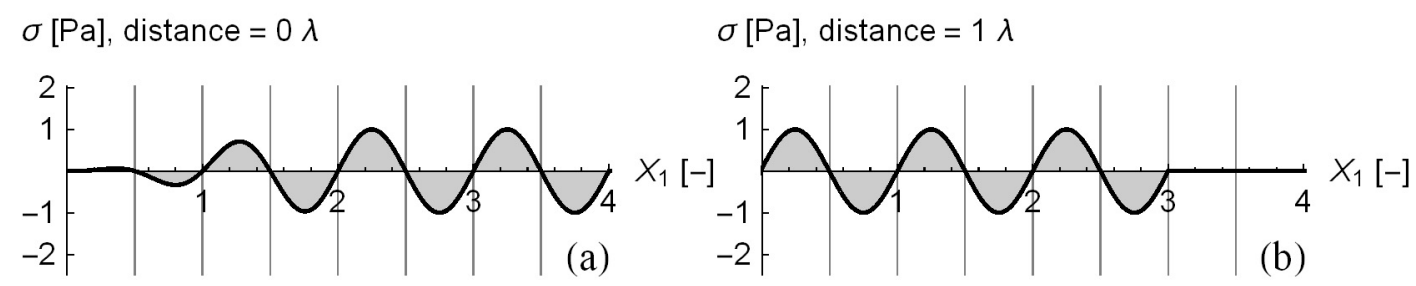

$\sigma[\mathrm{Pa}]$, distance $=2 \lambda$

$\sigma[\mathrm{Pa}]$, distance $=3 \lambda$

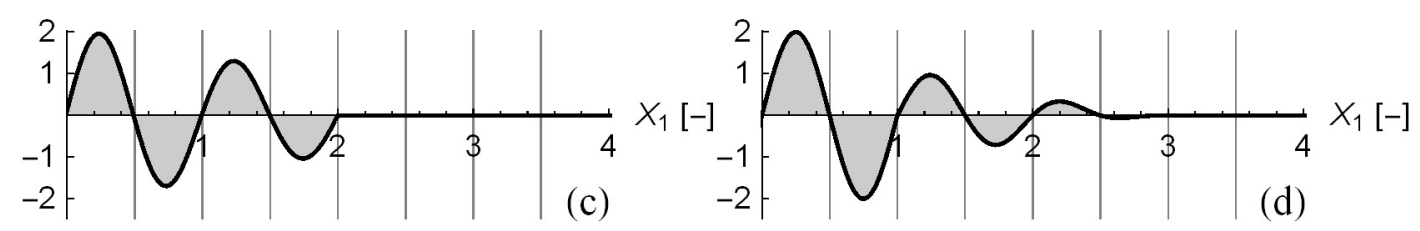

$\sigma[\mathrm{Pa}]$, distance $=4 \lambda$

$\sigma[\mathrm{Pa}]$, distance $=5 \lambda$
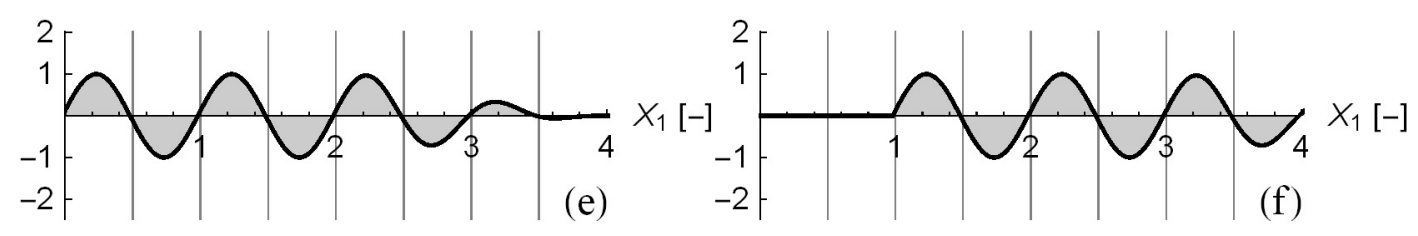

Fig. 3. The stress wave near the left free end of the $\operatorname{rod}\left(X_{1}=0\right)$ for various distances travelled by a wavefront, measured in wavelength $\lambda$ multiples (the incident wave (a) propagates to the left, the reflected wave (e) propagates to the right)

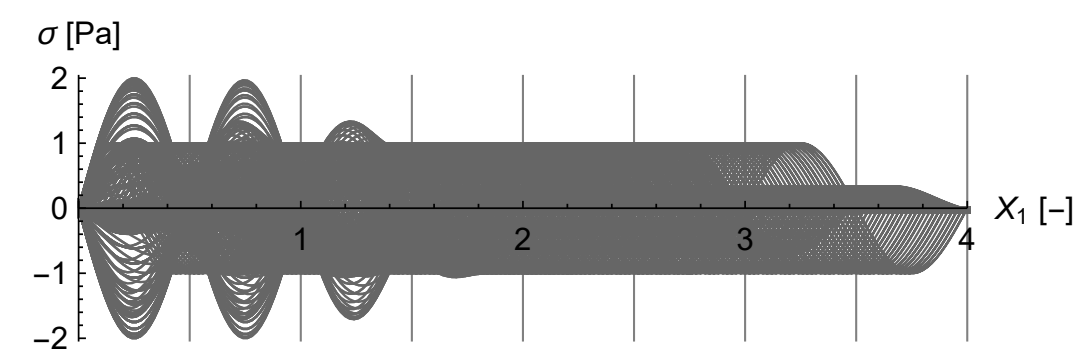

Fig. 4. Profile of the propagating wave packet, wavefront distance $X_{1}$ from 0 to 4.5 with a step of 0.015 (amplitude maxima are in positions $X_{1}=0.25,0.75$ and 1.25 from the left free end)

\subsection{Wave resulting from the impact of two rods}

The other possibility to excite a stress wave is a force pulse application generated, e.g., by the impact of a metal projectile on the rod free end. This case can be modelled using the elementary theory as a longitudinal impact of two elastic rods [7], see Fig. 5. The long rod I is at rest, the short rod II moves coaxially against the right end of the long rod at velocity $v$. We assume that the rods have the same cross-section $S$.

At the coaxial impact, the contact stress is developed at the boundary, which starts to propagate in both rods as a compressive stress wave at corresponding longitudinal wave velocities $c_{01}, c_{02}$. The stress wave in the impacting rod II arrives at its right end, reflects with the opposite amplitude, starts to subtract itself from the original stress and when the wavefront reaches the 

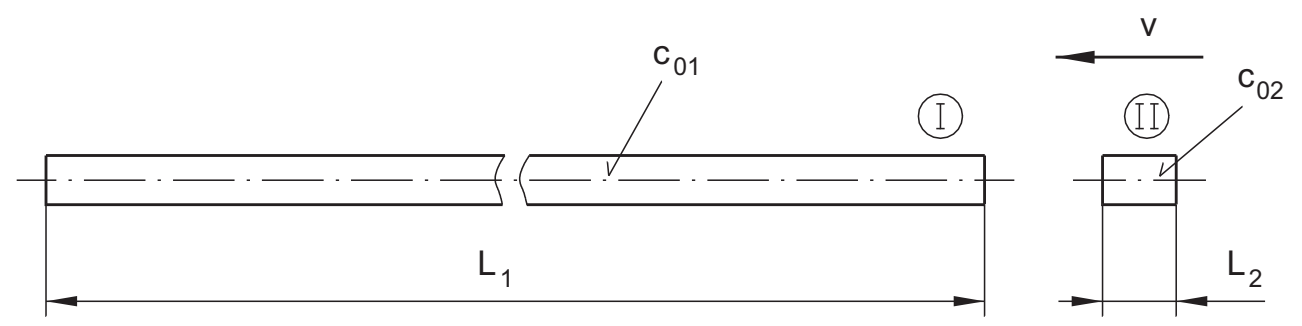

Fig. 5. Scheme of stress wave excitation by a longitudinal impact of two elastic rods

impact point, the stress in the entire rod is zero and the rod II has zero velocity. At this moment the pressure stops to act on the long rod I and the impact ends. The impact duration is therefore determined by a period of a waveform travel over two short rod lengths $2 L_{2}$.

At the same time the wavefront in the long rod reaches the distance $L_{p}=\frac{2 L_{2}}{c_{02}} c_{01}$ and the stress wave of length $L_{p}$ further propagates to the left end. The example of the theoretical square wave propagation near the rod left end is shown in Fig. 6. Similarly to Fig. 3, the horizontal axis is scaled in the relative displacement $X_{2}=c_{01} t / L_{p}$. The wave also reflects at the end, propagates back as a tensile stress and subtracts itself from the propagating compressive stress in the same way as in the short rod. The tensile wave starts to rise as lately as in the distance $L_{p} / 2$ from the rod end (see Fig. 6d) and from here it propagates in both directions. When the wave reaches the left rod end (Fig. 6e), it has already the final shape and propagates to the right impact point without changes. At the right end the wave reflects as a compressive wave and the whole process repeats.
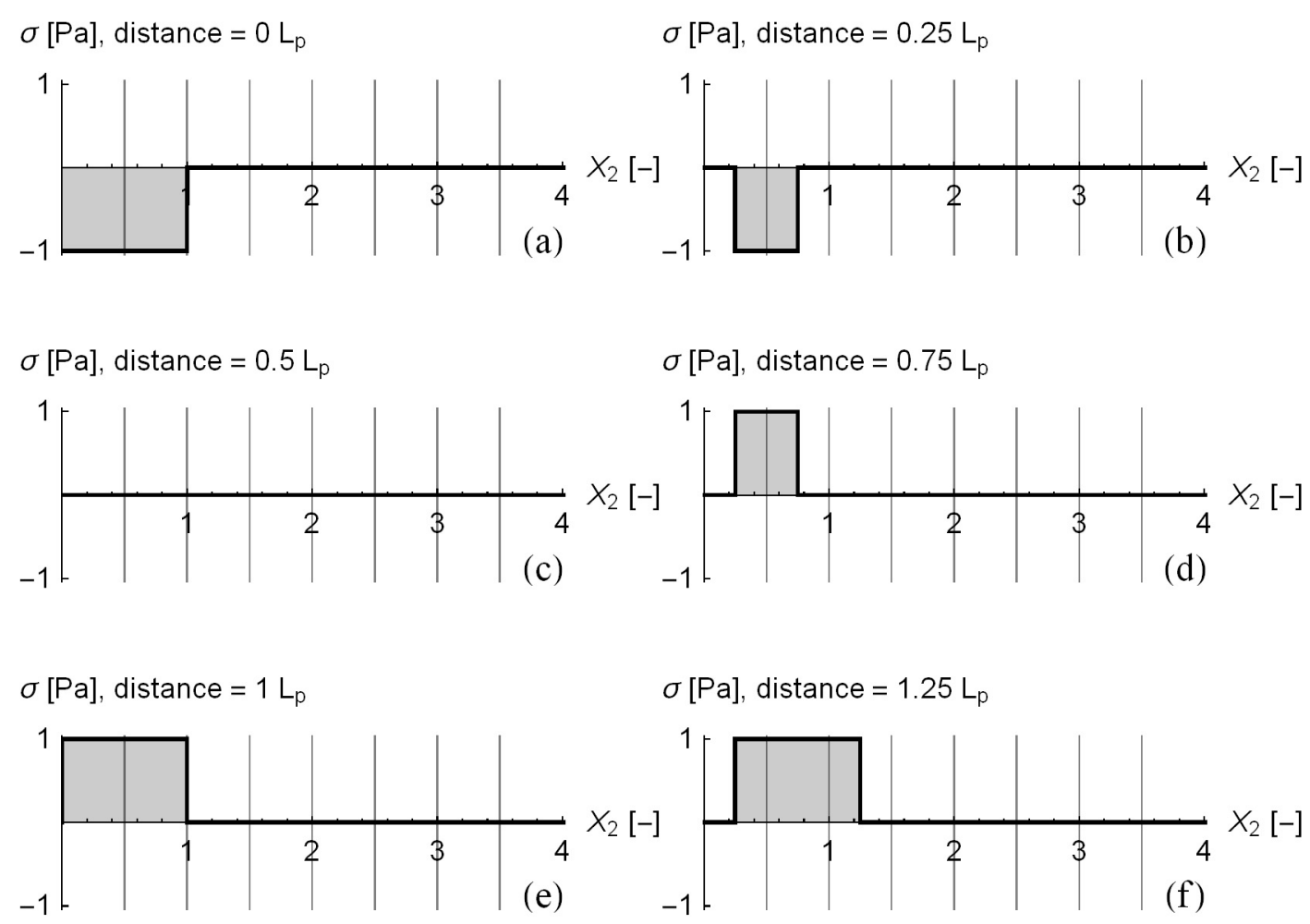

Fig. 6. The stress wave near the left free end of the rod $\left(X_{2}=0\right)$ for various distances travelled by a wavefront, measured in pulse length $L_{p}$ multiples (the incident wave (a) propagates to the left, the reflected wave (e) propagates to the right) 
The stress wave shape depends on the distance from the rod free end. At a position located more than $L_{p} / 2$, the stress is at first compressive for a period of $2 L_{2} / c_{02}$, and then after a certain delay is tensile for the same period. The delay between these opposite square waves is zero at the position $L_{p} / 2$ and the total stress wave has therefore double length. The length of the compressive and tensile wave parts is shorter at positions closer to the rod ends proportionally to the distance from the end until they vanish at the rod ends. Between individual waves, a zero stress interval exists, which is of length inversely proportional to the distance from the rod end (in the distance of $L_{p} / 2$ the interval is zero, at the end it is equal to the input wave length $L_{p}$ ). If the input wavelength $L_{p}$ is shorter than the rod length $L_{1}$, the shape of reflected stress waves is the same at the corresponding positions on both sides, only the phases are opposite.

For a long rod of brittle material having much higher compressive than tensile strength, the end section can be separated by virtue of the induced tensile stress after the wave reflection. For the theoretical square wave, the separation may occur in an arbitrary small distance from the end shorter than $L_{p} / 2$, but for a real wave with leading and trailing slopes (see, e.g., Fig. 10), the separation can occur only in a certain distance from the rod end.

The stress developed by this idealized rod impact is given by the relation $\sigma=v \frac{z_{1} z_{2}}{z_{1}+z_{2}}$, where $z_{1}=\rho_{1} c_{01}, z_{2}=\rho_{2} c_{02}$ are acoustic impedances of single rods. The induced stress is often high, e.g., by the impact of a steel rod on a glass rod at velocity $v=1 \mathrm{~m} / \mathrm{s}$, a stress of approximately $10 \mathrm{MPa}$ is developed in both materials.

\section{Measurement of waves in a glass rod}

The applicability of the separation technology was verified experimentally by performing impact tests on glass capillary tubes. The main objective of the measurements was the analysis of the wave propagation in the rod before and after an end separation. The stress waves were analyzed by means of the surface strain measurement using strain gauges. The dimensions of glass samples were $\varnothing 5.0 \mathrm{~mm} / \varnothing 0.9 \mathrm{~mm}$ diameter and $861 \mathrm{~mm}$ length. The measured material parameters include density $\rho=2490 \mathrm{~kg} / \mathrm{m}^{3}$ and wave velocity $c_{0}=5210 \mathrm{~m} / \mathrm{s}$, from which an approximate value of Young's modulus $E=6.7 \cdot 10^{10} \mathrm{~Pa}$ was obtained.

The scheme of the experimental setup is shown in Fig. 7. The samples were hung on thin wires at a horizontal position. The wave profile and the propagation behavior were measured by means of miniature resistance strain gauges (Micro-Measurements CEA-13-062UW-350)

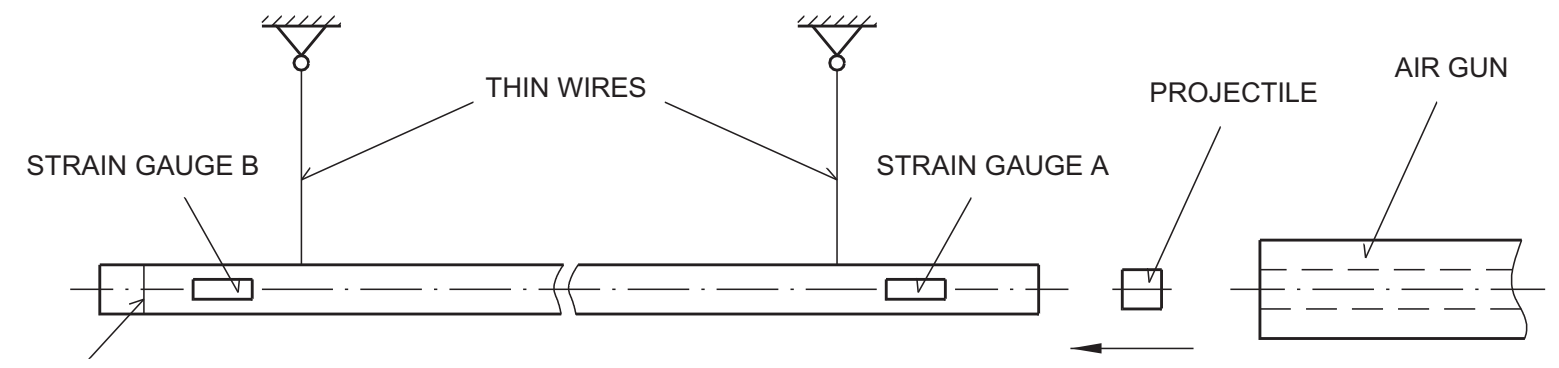

(NOTCH)

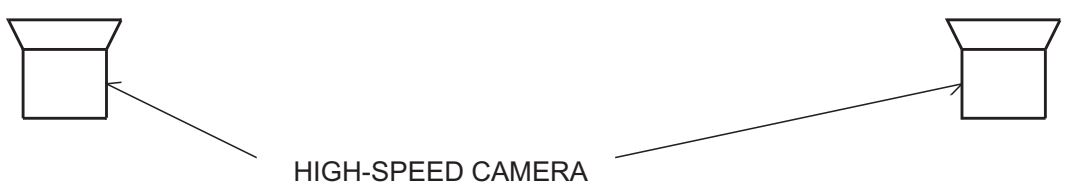

Fig. 7. Schematic diagram of the experimental setup 
bonded at A and B points near the rod ends. Strain gauge A was attached on the impact side at a distance of $67 \mathrm{~mm}$ from the end, on the opposite side strain gauge B was bonded at a distance of $65 \mathrm{~mm}$ from the free end. In these distances, the axial stress distribution over the cross-section is assumed to be uniform [5] (even in the case of end separation). Only one gauge was located at a measurement point and this configuration was sensitive also to a bending strain. The gauges were connected to DC bridges with $300 \mathrm{kHz}$ bandwidth. The signals were recorded by DEWE-5000 data acquisition system with a sampling frequency of $1 \mathrm{MHz}$. The strain gauge active length was only $1.7 \mathrm{~mm}$, therefore the wavefront travel time through the strain gauge is less than $0.4 \mu \mathrm{s}$. The local strain $\varepsilon$ in the rod axis direction was directly measured in non-dimensional units of $\mu$ strain $\left(10^{6} \Delta L / L\right)$. The stress in the rod in units of $\mathrm{Pa}$ can be calculated by multiplying the strain $\varepsilon$ by Young's modulus $E / 10^{6}$.

The stress waves in the rod were excited by an impact of a cylindrical lead projectile (a pellet) with a diameter of $4.5 \mathrm{~mm}$ (Fig. 8). The projectile was propelled by an air gun with helical grooving, which made it also rotate around horizontal axis. The impact velocity was lowered by a bored opening in the projectile axis. In addition to the strain gauges, the dynamic process was also captured by two high-speed cameras Olympus i-Speed at both rod ends with a frame rate of $10 \mathrm{kHz}$. The actual projectile velocity in the range from 70 to $100 \mathrm{~m} / \mathrm{s}$ was approximately derived from camera recordings using digital image processing.
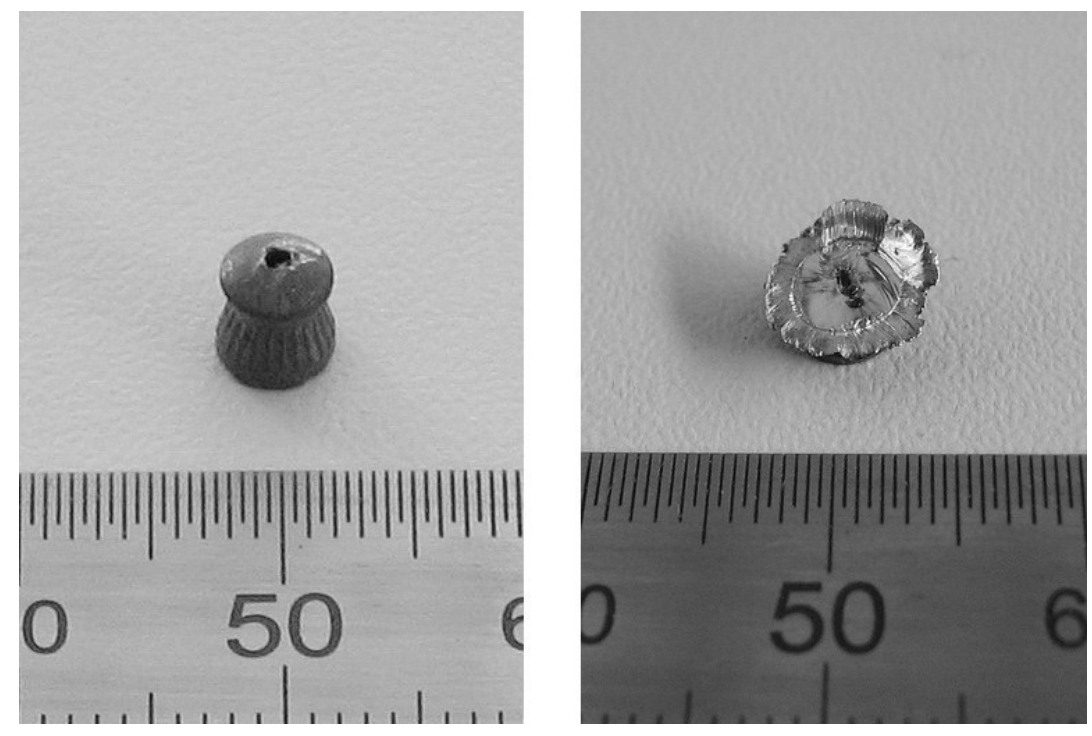

Fig. 8. Lead projectile (left - before impact, right - after impact)

\subsection{Notch-less rod}

Fig. 9 shows the strain histories at the strain gauges measurement points within approximately 46 wavefront travels along the rod length, the travelled distance is nearly $40 \mathrm{~m}$. The wave propagation repeats approximately after a wavefront travel over two rod lengths (after one cycle), however, the wave profile changes due to dispersion effects (wave attenuation and phase shift of frequency components) and the strain maxima gradually decrease.

Fig. 10 depicts the detailed impact time course, where the maximum compressive strain reaches $965 \mu$ strain. This corresponds to an impact force maximum to about $1300 \mathrm{~N}$. The positive part of the strain history at strain gauge A in Fig. 10 (i.e. tensile deformation) is most likely associated with the bending wave in the rod, excited by a small misalignment of 


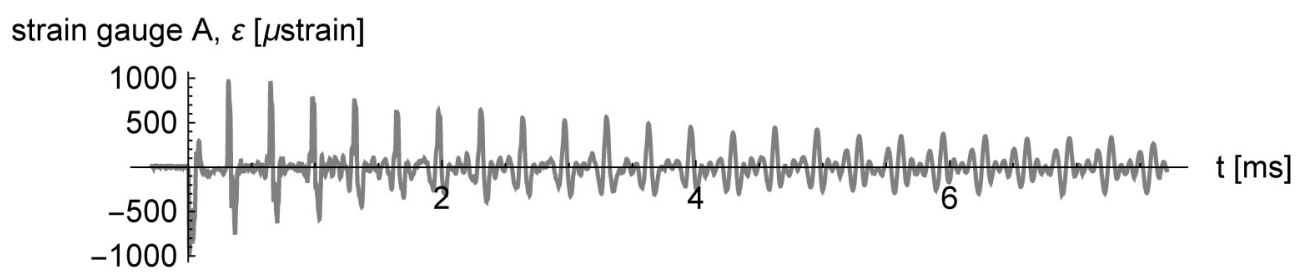

strain gauge $\mathrm{B}, \varepsilon[\mu$ strain $]$

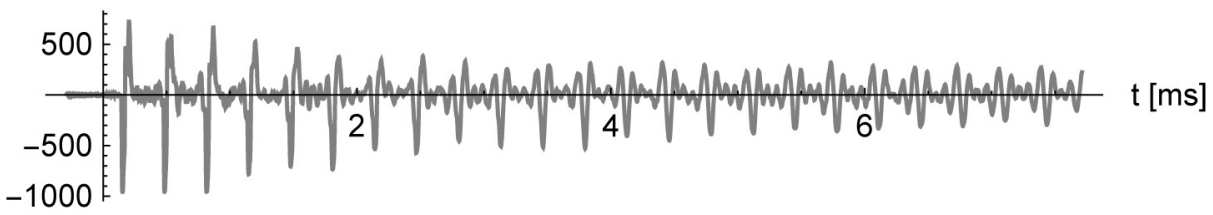

Fig. 9. Waves in the notch-less rod (approximately 46 wavefront runs along rod length)

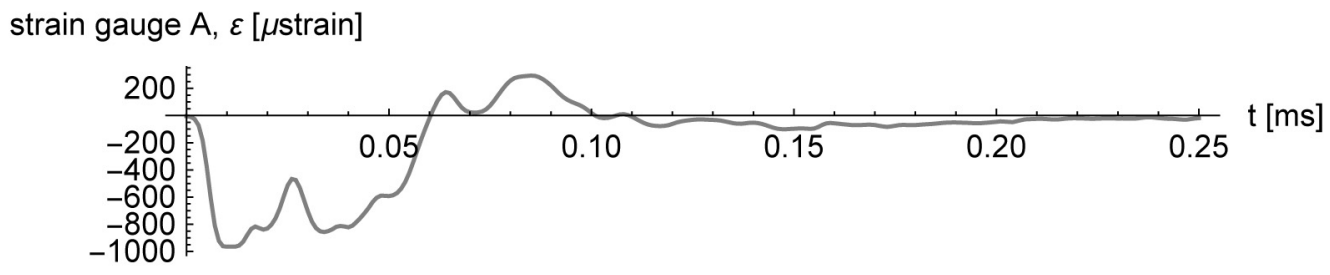

strain gauge $B, \varepsilon[\mu$ strain]

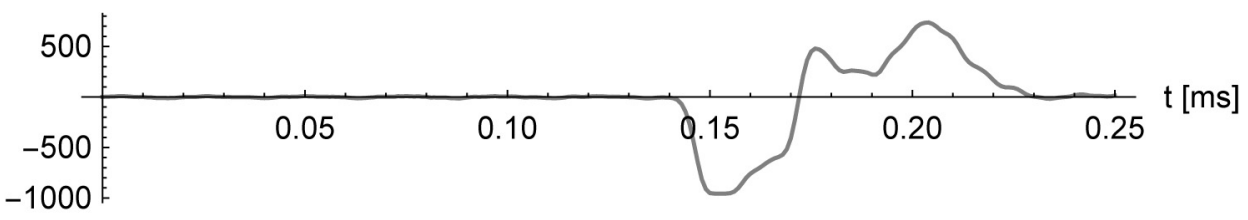

Fig. 10. Impact wave history at strain gauge A and following wave measured by strain gauge B

projectile and rod axes. The duration of measured compressive strain is nearly $60 \mu$ s and does not correspond to the assumed elastic wave propagation in the projectile. A relatively long length of wave $313 \mathrm{~mm}$ results from the projectile plastic deformation. Assuming that the stress is uniaxial, both elastic and plastic waves propagate at a velocity of $\sqrt{(1 / \rho)(d \sigma / d \varepsilon)}$, where $d \sigma / d \varepsilon$ is the variable Young's modulus. Mean modulus in the plastic region is approximately 70 times lower than in the elastic region for polycrystalline lead [13] (strain hardening of lead is very small) and the longitudinal wave velocity is hence circa 8.5 times lower. In this case, where the plastic deformation extends almost along the entire length of the projectile (see an example in Fig. 8), the elastic waves in the projectile have a little effect on the impact wave length. The projectile shape is complicated and the development of its deformation is complex. The actual projectile velocity at this measurement was about $90 \mathrm{~m} / \mathrm{s}$. The measured wave duration approximately corresponds to these considerations.

From the wave history at gauge A (Fig. 10 top), the initial pulse course can be derived and then the propagation and the reflections of this wave and the strain time courses in the arbitrary rod positions can be calculated using (7) and (10). Only the compressive part of the input wave was considered for calculations (the tensile part resulting almost certainly from the bending wave, which has a different propagation behavior, is neglected). In Fig. 11, the theoretical results (grey) are compared with the measurement (black) at both gauge points. A one-dimensional model assumes a steady-state wave propagation without distortion, however, 


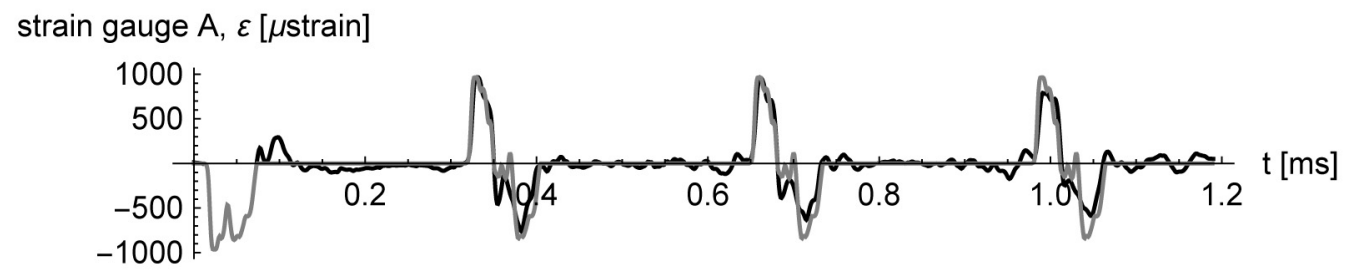

strain gauge $B, \varepsilon[\mu$ strain]

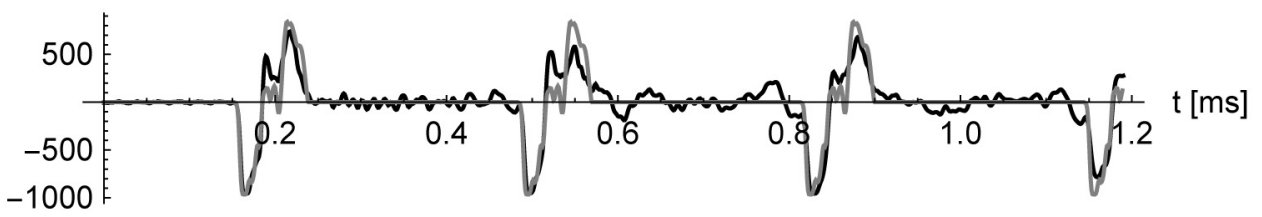

Fig. 11. Comparison of the measured (black) and the calculated (gray) wave history at strain gauges A and $\mathrm{B}$ measurement points

the influence of the wave dispersion on the pulse shape is evident. The difference between the calculated and the measured wave increases with the reflection number, whereas the leading wave parts always agree better with the measurement. Nevertheless, the accuracy of model results is sufficient for the short-time analysis of the wave propagation before and after the first reflection.

\subsection{Rod with circumferential notch}

During tests with capillary tubes having a circumferential notch in $15 \mathrm{~mm}$ distance from the end, the preforms were separated by the tensile stress wave at the notch position. As can be seen from Fig. 12, the measured strain history is similar to the case without separation, however, the frequency of wave reflections increased after the first reflection due to rod truncation caused by end separation. The projectile velocity was $75 \mathrm{~m} / \mathrm{s}$ in this case.

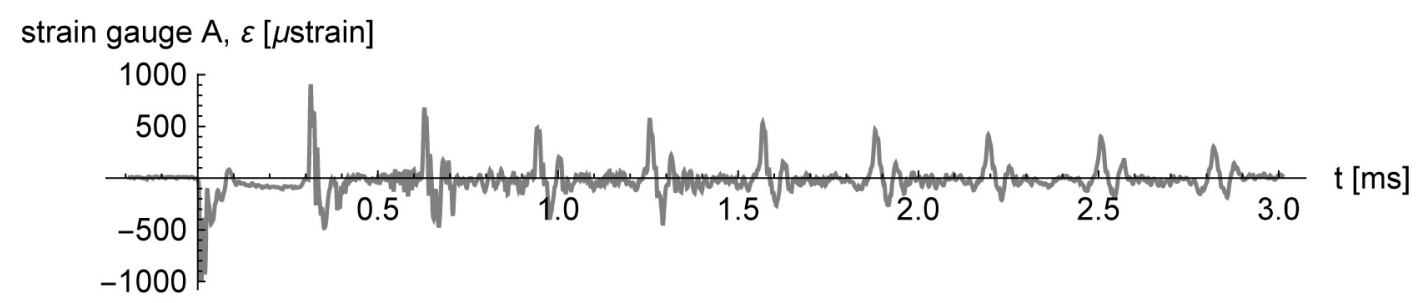

Fig. 12. Waves in rod after end separation

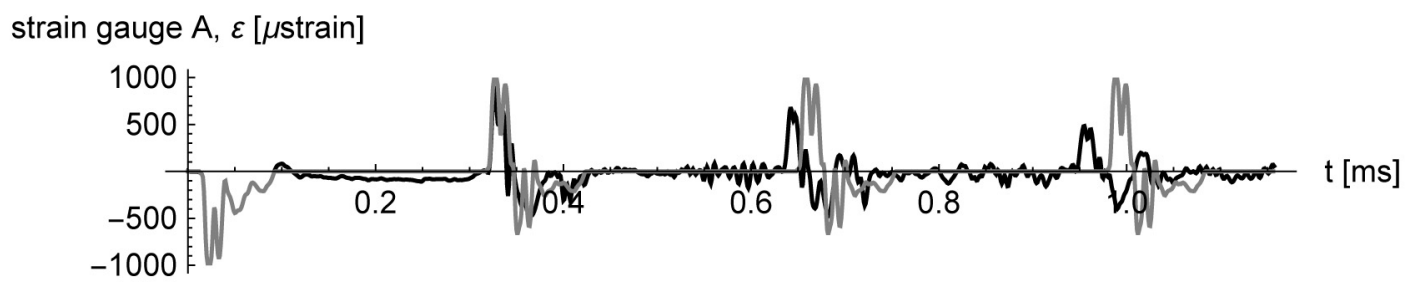

Fig. 13. Comparison of the measured (black) and the calculated (gray) wave history at strain gauge A measurement point after end separation

The detailed comparison of the measured and the calculated wave history is given in Fig. 13. The initial pulse measured by strain gauge A was used for the calculation again (see Fig. 12, 
only the impact compressive part was considered). Similar to the previous case, the theoretical wave propagates uniformly, so that the end separation effect and the wave dispersion are clearly seen in this figure. It is obvious that the end separation does not significantly influence the first reflected wave travelling through strain gauge A. In the next passage the measured wave (black) arrives earlier due to a rod truncation and this also corresponds to a higher frequency of reflections. The decrease of the wave maxima is greater than in the case without a separation (Fig. 9). The reason is mainly the energy loss during the end separation.

A number of rod-cutting tests were performed with various impact velocities in the range from 70 to $100 \mathrm{~m} / \mathrm{s}$. The fracture surface quality was varying and it was mostly influenced by the impact parameters (velocity, alignment of projectile and rod axes) and by the hand-cut notch quality. The cutting test conditions were not perfectly adjustable and the deformable lead projectile was not optimal. The cutting quality and the repeatability should be improved by the intended machine automation. Fig. 14 shows an example of sufficient high-quality surface fracture after the capillary end separation.

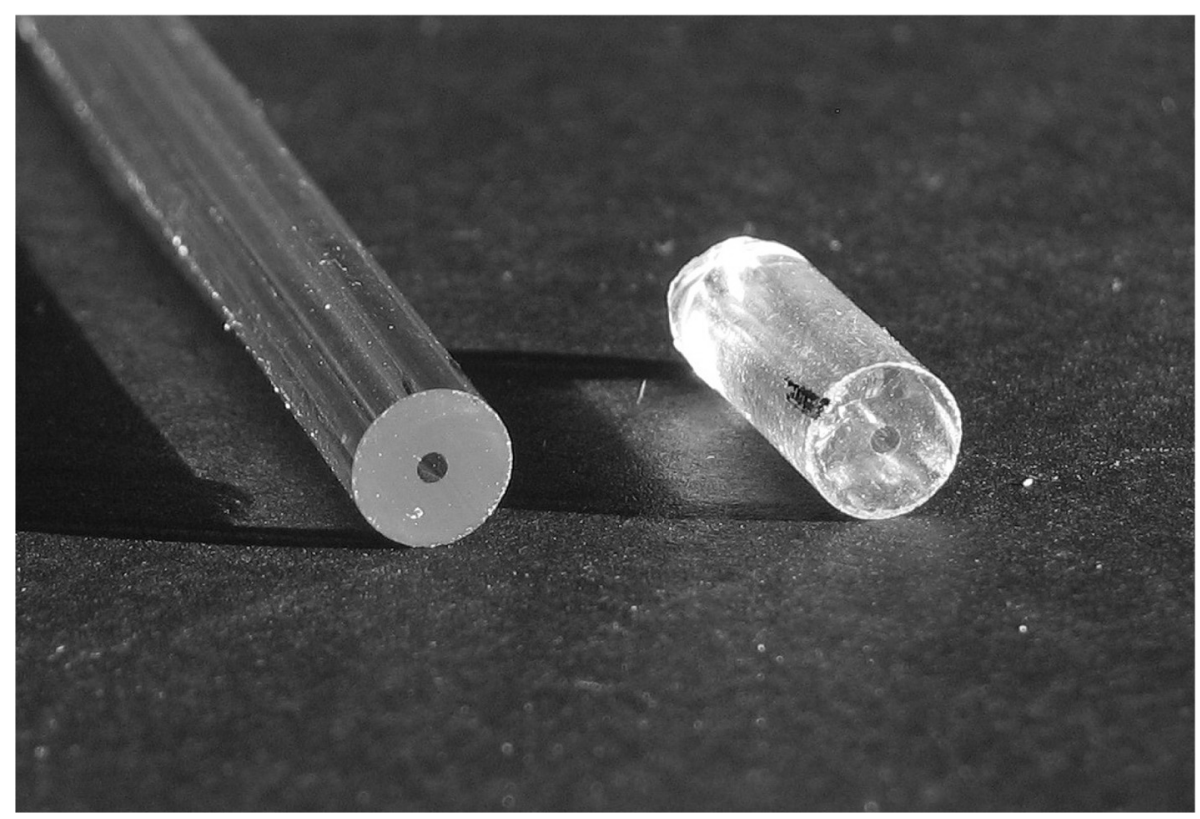

Fig. 14. Example of fracture surface after end separation

\section{Conclusion}

An innovative technology for cutting brittle notched rods was recently developed, which is based on a longitudinal stress wave propagation followed by a reflection from a rod free end. Since the tensile strength of brittle materials is much lower than the compressive strength, the separation of the notched end may occur by virtue of the tensile stress. The main concern of the paper was to study the basic characteristics and the applicability of this separation method using the theoretical and the experimental analysis.

The one-dimensional theory for thin elastic rods was used for the description of the stress wave propagation. Assuming the short-time analysis of the wave motion and neglecting all the dispersion effects, this model was sufficient for the initial study. The propagation of two potential stress shapes (harmonic wave packet and pulse wave) were simulated. For practical application, the pulse wave resulting from the impact of two rods proved to be more suitable. 
The experimental assessment of the wave propagation before and after the rod end separation was carried out by performing impact tests on glass capillary tubes. The stress waves were analyzed by means of the surface strain measurement using strain gauges. The measured wave histories were compared with the theoretical model to study the influence of the dispersion and the end separation effects. The preform separation at the notch position was successfully achieved for tubes with a circumferential notch. This confirms a future applicability of this technology for cutting brittle rods. The cutting test conditions were not perfectly adjustable and the lead projectile used for the impact excitation was not optimal, which resulted in a varying quality of the fracture surface. The results presented in the paper form the basis for a more detailed research in this field. The intended machine automation and a suitable modification of the initial pulse shape should improve the cutting quality and the repeatability.

\section{Acknowledgements}

This work was supported by the Czech Ministry of Industry and Trade in the framework of the institutional support for the long-term conceptual development of the research organization.

\section{References}

[1] Benatar, A., Rittel, D., Yarin, A. L., Theoretical and experimental analysis of longitudinal wave propagation in cylindrical viscoelastic rods, Journal of the Mechanics and Physics of Solids 51 (8) (2003) 1413-1431. https://doi.org/10.1016/S0022-5096(03)00056-5

[2] Bracewell, R. N., The Fourier transform and its applications, McGraw Hill, Singapore, 2000.

[3] Brepta, R., Strain waves and impact phenomenona in linear-elastic and viscoelastic media, TU of Liberec, LENAM, Liberec, 1997 (in Czech).

[4] Diaz-Rubio, F. G., Perez, J.R., Galvez, V.S., The spalling of long bars as a reliable method of measuring the dynamic tensile strength of ceramics, International Journal of Impact Engineering 27 (2) (2002) 161-177. https://doi.org/10.1016/S0734-743X(01)00039-2

[5] Gama, B. A., Lopatnikov, S. L., Gillespie Jr., J. W., Hopkinson bar experimental technique: A critical review, Applied Mechanics Reviews 57 (4) (2004) 223-250. https://doi.org/10.1115/1.1704626

[6] Graff, K. F., Wave motion in elastic solids, Dover Publications, New York, 1991.

[7] Höschl, C., Impact strength of solids, Dům techniky ČSVTS, Praha, 1977 (in Czech).

[8] Klepaczko, J.R., Brara, A., An experimental method for dynamic tensile testing of concrete by spalling, International Journal of Impact Engineering 25 (4) (2001) 387-409. https://doi.org/10.1016/S0734-743X(00)00050-6

[9] Li, T., Chen, Y., Ma, J., Development of a miniaturized piezoelectric ultrasonic transducer, IEEE Transactions on Ultrasonics, Ferroelectrics, and Frequency Control 56 (3) (2009) 649-659. https://doi.org/10.1109/TUFFC.2009.1081

[10] Menčík, J., Strength and fracture of glass and ceramics, Elsevier, Amsterdam, New York, 1992.

[11] Niu, L., Zhu, W., Li, S., Liu, X., Spalling of a one-dimensional viscoelastic bar induced by stress wave propagation, International Journal of Rock Mechanics and Mining Sciences 131 (2020) 104317. https://doi.org/10.1016/j.ijrmms.2020.104317

[12] Pustka, M., Šidlof, P., Erhart, J., Method for separating a rod-like or tubular object made of a brittle material, European patent specification EP3480172B1, 2020.

[13] Shea, J. H., Propagation of plastic strain pulses in cylindrical lead bars, Journal of Applied Physics 39 (8) (1968) 4004-4011. https://doi.org/10.1063/1.1656889

[14] Weisstein, E. W., Polylogarithm, MathWorld - A Wolfram Web Resource, 2020. http://mathworld.wolfram.com/Polylogarithm.html 